\title{
Optical identifications of high-redshift galaxy clusters from Planck Sunyaev-Zeldovich survey
}

\author{
R.A. Burenin" ${ }^{* 1}$, I.F. Bikmaev ${ }^{2,3}$, I.M. Khamitov ${ }^{2,4}$, I.A. Zaznobin ${ }^{1}$, \\ G.A. Khorunzhev ${ }^{1}$, M.V. Eselevich ${ }^{5}$, V.L. Afanasyev ${ }^{6}$, S.N. Dodonov ${ }^{6}$, \\ J.A. Rubiño-Martín ${ }^{7}$, N. Aghanim ${ }^{8}$, R.A. Sunyaev ${ }^{1,9}$ \\ ${ }^{1}$ Space Research Institute RAS, Moscow, Russia \\ ${ }^{2}$ Kazan Federal University, Kazan, Russia \\ ${ }^{3}$ Academy of Sciences of The Republic of Tatarstan, Kazan, Russia \\ ${ }^{4}$ Observatory of Scientific and Technological Research Council of Turkey, Antalya, Turkey \\ ${ }^{5}$ Institute of Solar-Terrestrial Physics SB RAS, Irkutsk, Russia \\ ${ }^{6}$ The Special Astrophysical Observatory of the Russian Academy of Sciences, Nizhnij Arkhyz, Russia \\ ${ }^{7}$ Instituto de Astrofísica de Canarias, Tenerife, Spain \\ ${ }^{8}$ Institut d'Astrophysique Spatiale, Orsay, France \\ ${ }^{9}$ Max Planck Institute for Astrophysics, Garching, Germany
}

Received January 16, 2018

\begin{abstract}
We present the results of optical identifications and spectroscopic redshifts measurements for galaxy clusters from 2-nd Planck catalogue of Sunyaev-Zeldovich sources (PSZ2), located at high redshifts, $z \approx 0.7-0.9$. We used the data of optical observations obtained with Russian-Turkish 1.5-m telescope (RTT150), Sayan observatory 1.6-m telescope, Calar Alto 3.5-m telescope and 6-m SAO RAS telescope (Bolshoi Teleskop Alt-azimutalnyi, BTA). Spectroscopic redshift measurements were obtained for seven galaxy clusters, including one cluster, PSZ2 G126.57+51.61, from the cosmological sample of PSZ2 catalogue. In central regions of two clusters, PSZ2 G069.39+68.05 and PSZ2 G087.39-34.58, the strong gravitationally lensed background galaxies are found, one of them at redshift $z=4.262$. The data presented below roughly double the number of known galaxy clusters in the second Planck catalogue of Sunyaev-Zeldovich sources at high redshifts, $z \approx 0.8$.
\end{abstract}

Key words: galaxy clusters, sky surveys

\section{INTRODUCTION}

In Planck all-sky survey, by means of the observations of Sunyaev-Zeldovich effect (SZ, Sunyaev and Zeldovich, 1972), the most massive clusters in observable Universe are detected more or less uniformly over the entire sky (Planck Collaboration, 2014b, 2016c). This sample is unique and is of great interest for various cosmological studies such as obtaining the cosmological parameter constraints using the measurements of the galaxy cluster mass function (e.g. Vikhlinin et al., 2009a,b; Burenin and Vikhlinin, 2012; Planck Collaboration, 2014a, 2016b). In particular, virtually all the clusters discovered in Planck SZ survey will be included into the large cosmological samples of massive galaxy clusters that will be selected in the future Spectrum-Roentgen-Gamma (SRG) / eROSITA all-sky Xray survey.

The second Planck Sunyaev-Zeldovich sources catalogue, released in 2015 (PSZ2, Planck Collaboration, 2016c), contains 1653 objects of which 1203 are confirmed

\footnotetext{
*e-mail: rodion@hea.iki.rssi.ru
}

as massive galaxy clusters. Among the unidentified SZ sources there is some number of false detections, but also there is a significant number of unidentified galaxy clusters. Our group take part in the work on optical identifications and redshift measurements of the galaxy clusters from this survey (Planck Collaboration, 2014b, 2015a,b, 2016c,d; Vorobiev et al., 2016; Burenin et al., 2016; Burenin, 2017; Amodeo et al., 2017).

The completeness of optical identification of SunyaevZeldovich sources in Planck survey changes with redshift. Nearby rich clusters located at redshifts below $z \approx 0.3$ currently are well studied and, in the vast majority, were known before Planck survey was started. Clusters at higher redshifts, up to $z \approx 0.7$, can be detected in the Sloan Digital Sky Survey (SDSS) images and also they can be quite easily observed with modern 1.5-3-m telescopes. Therefore, the completeness of the optical identification of clusters at such redshifts is high (Planck Collaboration, 2014b, 2015b, 2016c).

The optical identification of more distant clusters is generally much more complicated, so the completeness of the 
Planck catalog clusters should be lower. In this paper we present the results of our systematic search for the distant galaxy clusters among the objects from second catalogue of Planck SZ sources, using the data in optical and near IR. We present the data on the optical identification and spectroscopic redshift measurements of seven galaxy clusters at $z>0.7$. These data roughly doubles the number of known galaxy clusters in the second catalogue of Planck SZ sources at these high redshifts.

\section{OBJECT SELECTION}

Preliminary selection of objects was done using the data of WISE (Wright et al., 2010), SDSS (SDSS Collaboration, 2017) and Pan-STARRS1 (Chambers et al., 2016) sky surveys. We used the images from WISE survey in $3.4 \mu \mathrm{m}$ band, where all the stars in the field were subtracted using WISE PSF model, while the stars were identified using SDSS data. WISE images cleaned from stars were then smoothed using $\beta$-model with $24^{\prime \prime}$ radius (which corresponds to the radius of $180 \mathrm{kpc}$ at $z=0.8$ ). In these images clusters of galaxies can be identified up to high redshifts $z \approx 1-2$, and their IR luminosity correlates well with cluster mass (e.g., Burenin, 2015, 2017).

Clusters located at redshifts below $z \approx 0.6$ can be identified using the data of SDSS survey (Rykoff et al., 2014), using additional data from WISE all-sky survey it is possible to identify galaxy clusters at higher redshifts, up to $z \approx 0.7$ (Burenin, 2017). To identify clusters at even higher redshifts, deeper direct imaging data in red and near IR bands are required. In our work for these purposes was used data of the Pan-STARRS1 survey and also the observations at various optical telescopes (see below).

It turns out that in Pan-STARRS1 images only the brightest galaxies in clusters at redshifts $z \approx 0.7-0.9$ can be detected. However, from the colors of these galaxies is possible to obtain a rough estimate of their redshift. In Fig. 1 the relation between the median color $i-z$ of the brightest galaxies in clusters and cluster redshifts is shown. The list of high-redshift galaxy clusters here are taken from the ROSAT 400 square degree X-ray cluster survey (Burenin et al., 2007) and from PSZ2 catalogue. The red line shows the color of synthetic stellar population spectrum with 11 Gyear age and $Z=0.02$ metallicity taken from Bruzual and Charlot (2003). One can see that using these data it is possible to obtain a photometric redshift estimate with the accuracy not worse than approximately $\delta z /(1+z) \approx 0.03$, in $0.7<z<0.9$ redshift range.

Using these data, 18 objects from PSZ2 catalogue were selected. Three of them were discarded on the basis of deep direct images of these fields (see below), observations of eight objects will be continued. Seven Sunyaev-Zeldovich sources were identified with high-redshift galaxy clusters, located at redshifts above $z \approx 0.7$. For the brightest galaxies in these clusters optical spectra were obtained, which

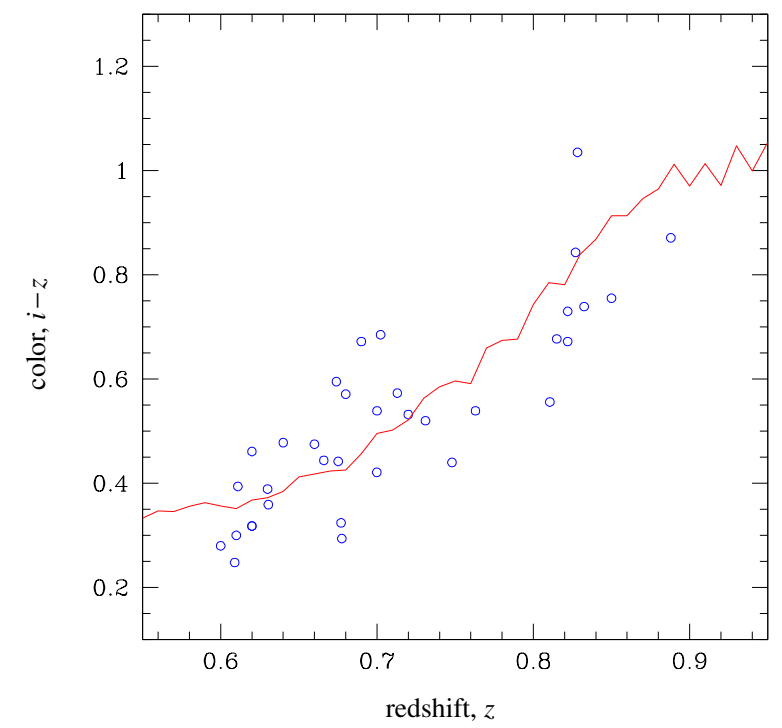

Fig. 1. The relation between cluster redshifts and median $i-z$ colors of the brightest cluster galaxies measured using Pan-STARRS1 photometric data. The red line shows the color of synthetic stellar population spectrum with 11 Gyear age and $Z=0.02$ metallicity taken from Bruzual and Charlot (2003).

allowed to measure cluster spectroscopic redshifts. These the observations are discussed in detail below.

\section{OBSERVATIONS}

Imaging and spectroscopic observations of objects selected as high-redshift, $z>0.7$, galaxy cluster candidates were made at various telescopes available to our group. Deep direct images in SDSS filters $r i$ were obtained with 1.5-m Russian-Turkish telescope (RTT150) using the TFOSC ${ }^{1}$ instrument, with 6-m telescope of SAO RAS (Bolshoi Telescop Alt-azimuthalny, BTA-6m) using SCORPIO and SCORPIO-2 instruments (Afanasiev and Moiseev, 2005, 2011), and also at the Calar Alto 3.5-m

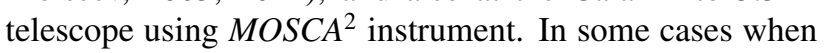
SDSS $r i$ filters were not available, Bessel $R I$ filters were used.

The exposures were chosen to reach the magnitude limits not worse than about $25^{m}$ in $r$ and $24^{m}$ in $i$ bands $(5 \sigma)$. This required the 1-2 $\mathrm{h}$ exposures at 1.5-m telescope RTT150, 15-30 min exposures at Calar Alto 3.5-m telescope and 5$10 \mathrm{~min}$ at BTA 6-m telescope. All of these the data were obtained at seeing not worse than $1.5^{\prime \prime}$. The total exposure was split into few smaller exposures so that in each image the number of background counts does not exceed a few thousands ADU. Between the exposures the telescope pointing was shifted by $10-20^{\prime \prime}$. During the data reduction the images were corrected for bias and flat field, the images in $i, I$ bands were further corrected for fringes. The images

\footnotetext{
${ }^{1}$ http://hea.iki.rssi.ru/rtt150/en/index.php?page=tfosc

${ }^{2}$ http://w3.caha.es/CAHA/Instruments/MOSCA/
} 


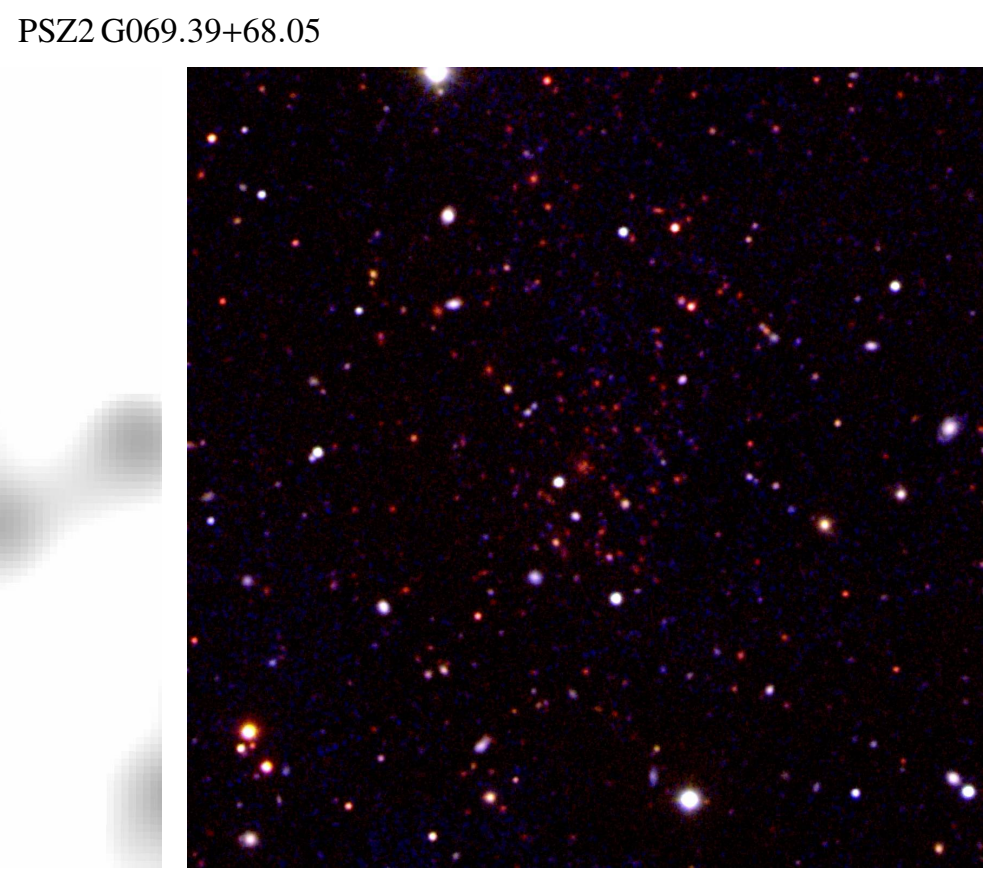

PSZ2 G087.39-34.58
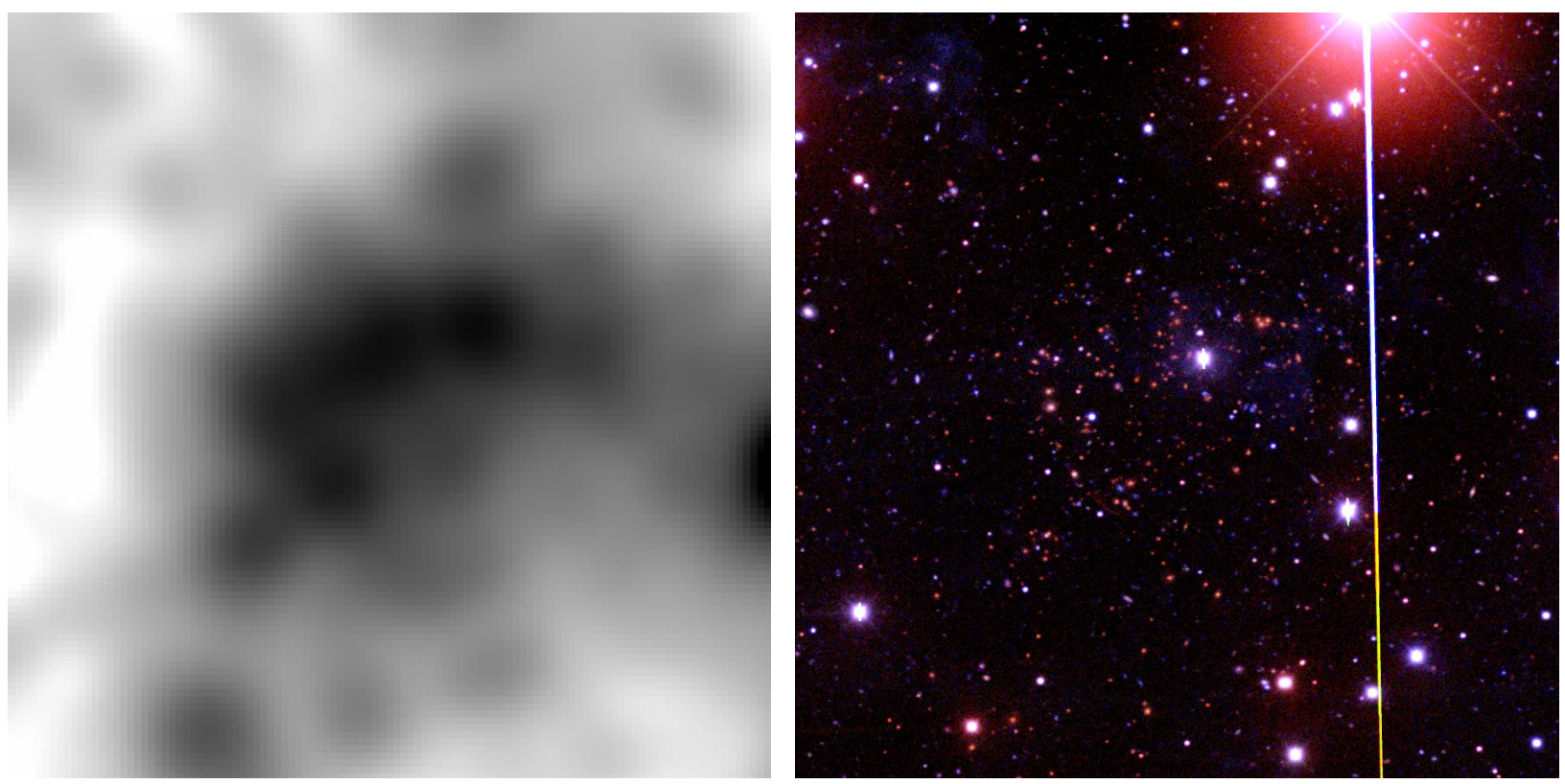

Fig. 2. - Left: the image in $3.4 \mu \mathrm{m}$ band from WISE survey, cleaned from stars and convolved with $\beta$-model of $24^{\prime \prime}$ radius. Right: pseudocolor image of the field in SDSS irg filters $(R G B)$. The images are centered at the clusters optical centers, the image size is $5^{\prime} \times 5^{\prime}$. 

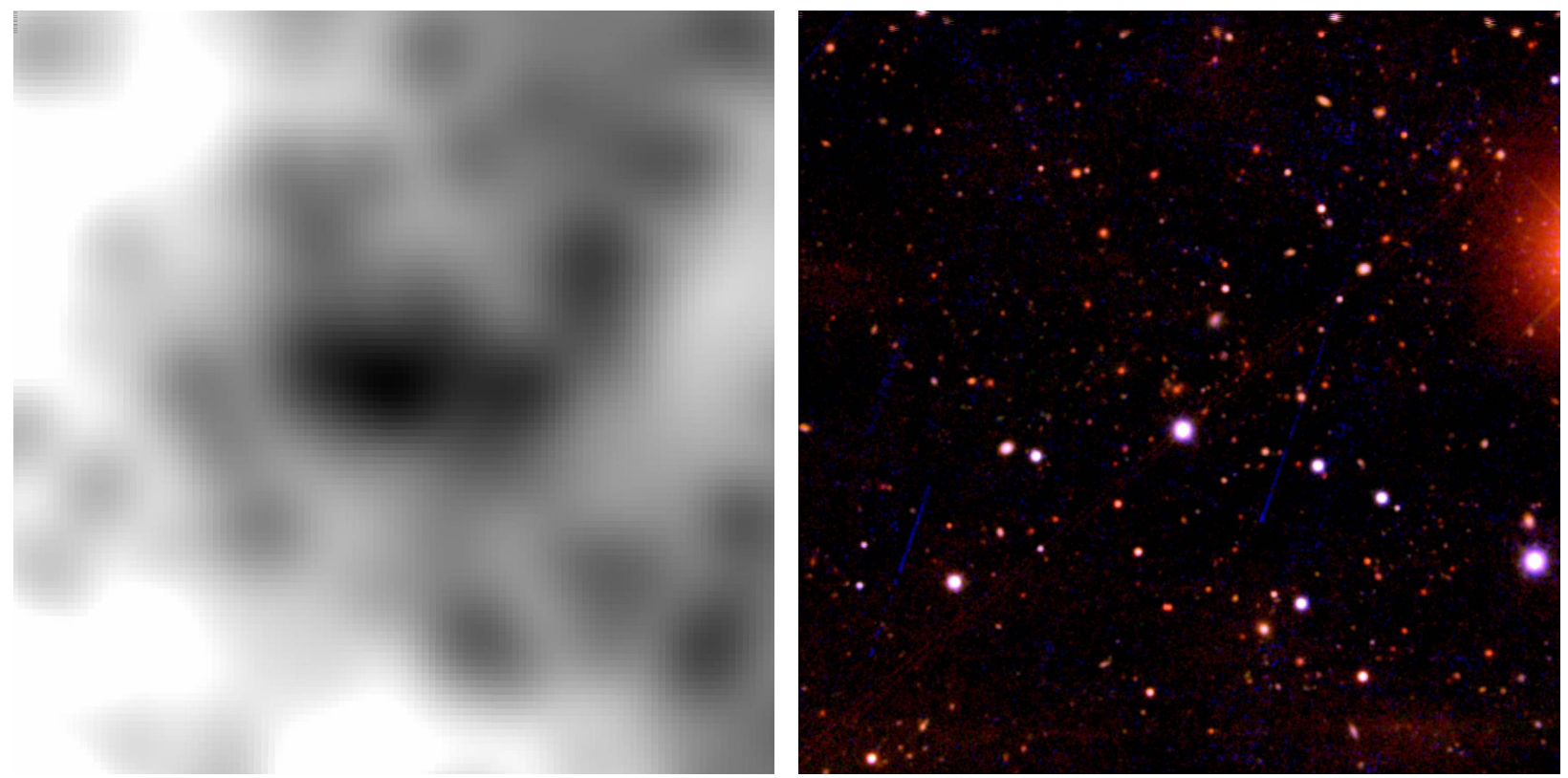

PSZ2 G126.28+65.62
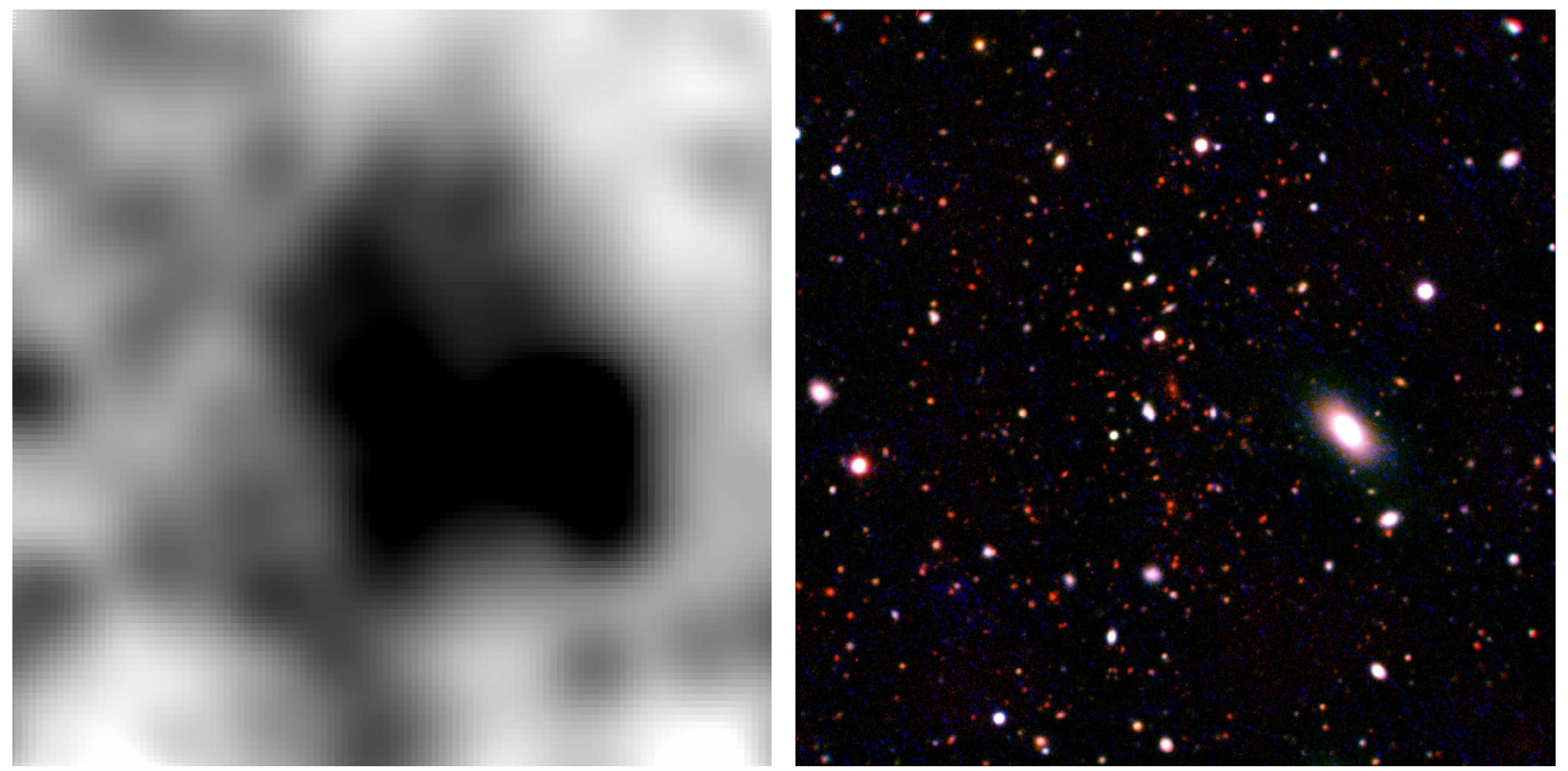

Fig. 2. - Continued. 


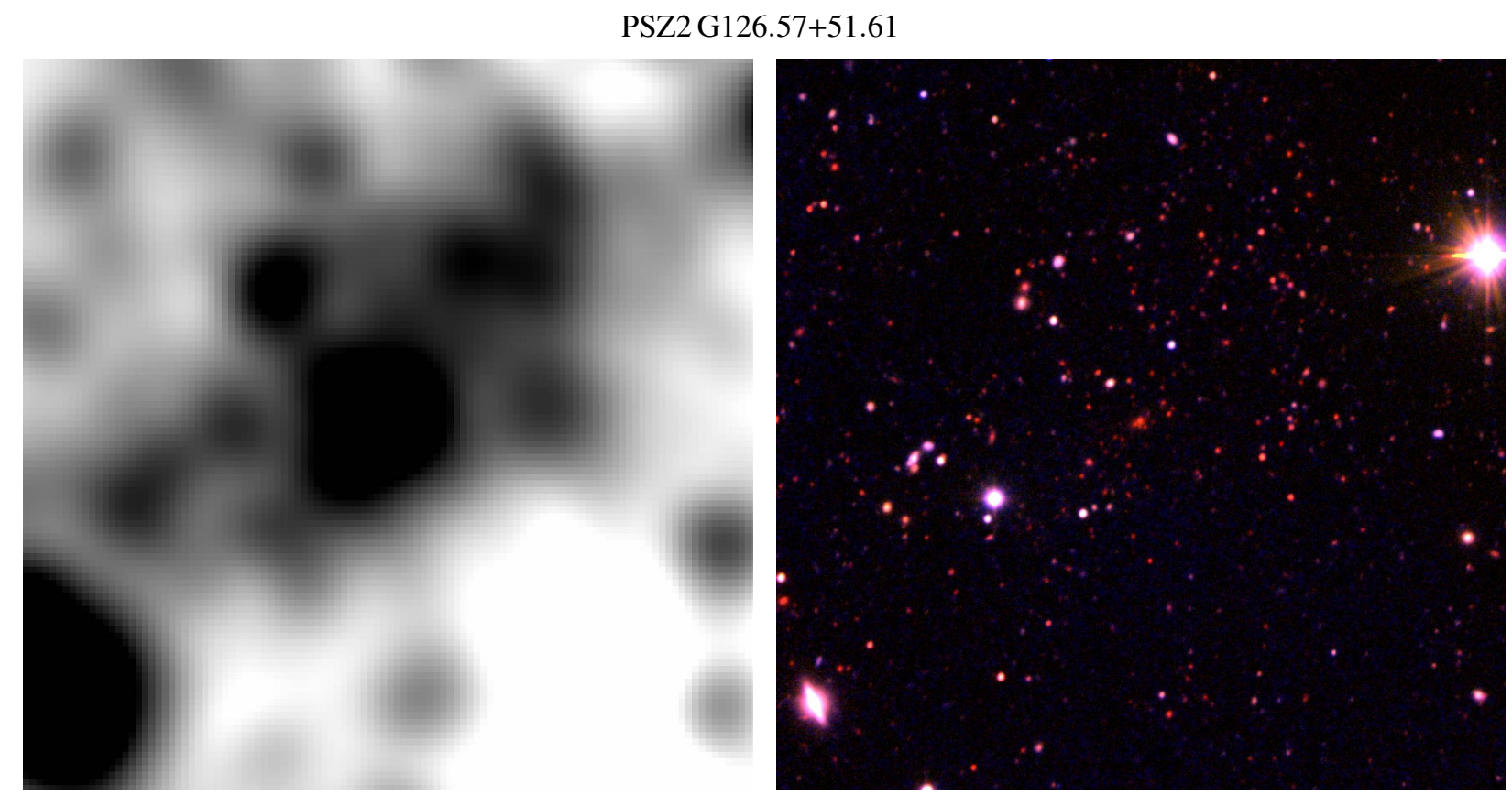

PSZ2 G237.68+57.83

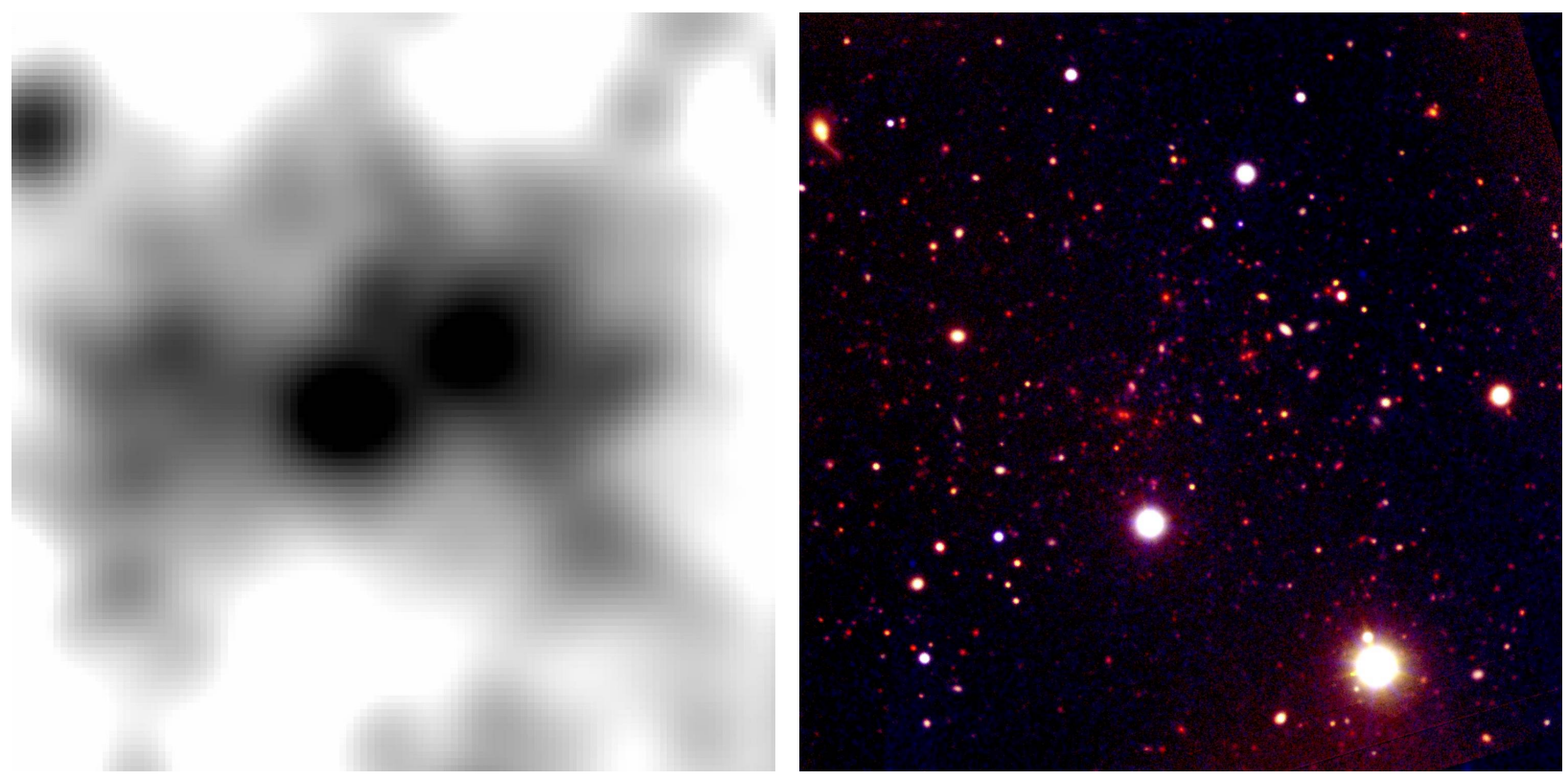

Fig. 2. - Continued. 


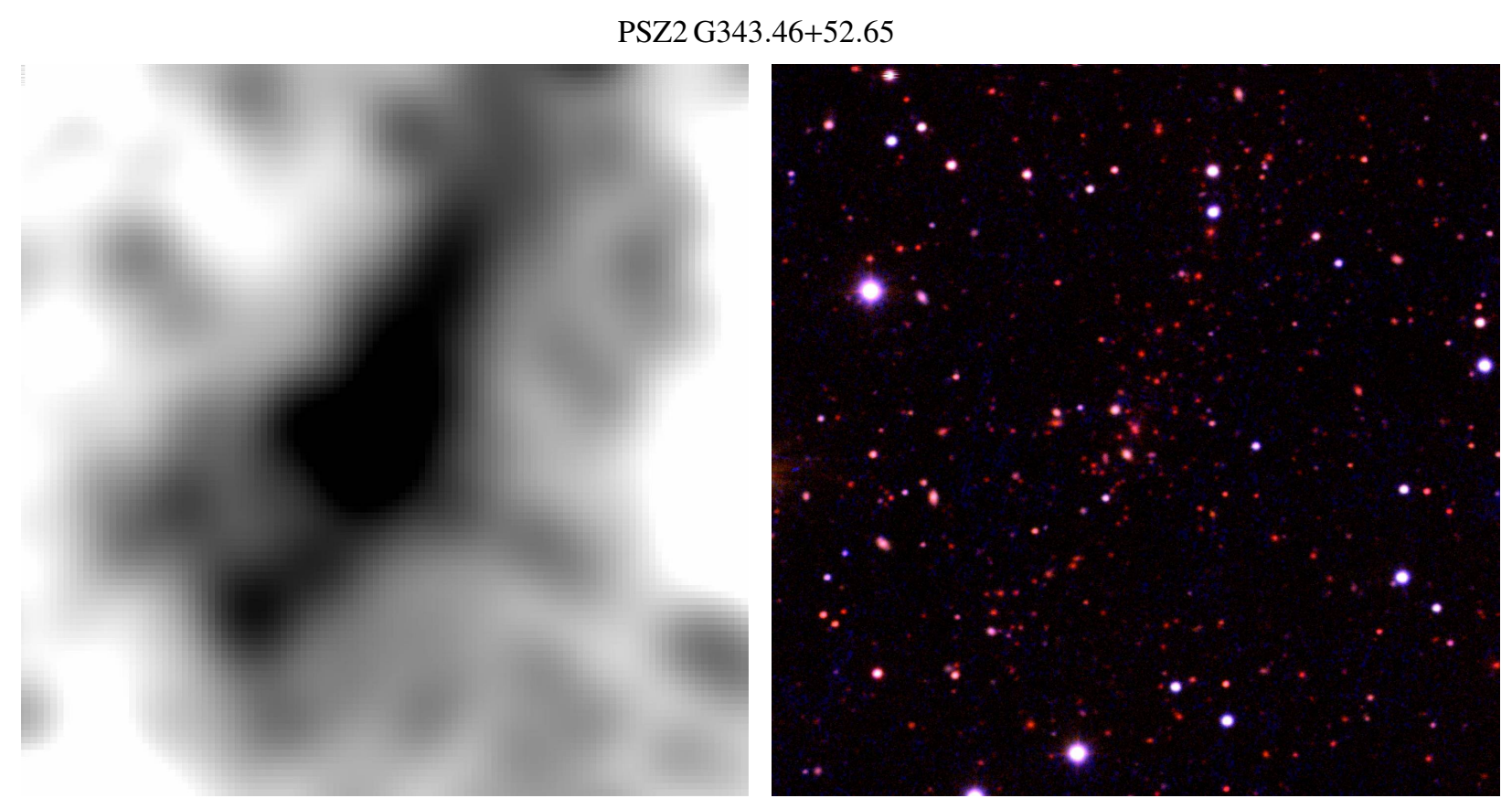

Fig. 2. - Continued.

were then aligned and combined using the algorithms allowing to remove cosmic rays and to reduce systematic errors of flat field calibrations.

The spectra of brightest cluster galaxies were obtained mainly at BTA 6-m telescope using SCORPIO and SCORPIO-2 instruments (Afanasiev and Moiseev, 2005, 2011). The spectrum of one object, the brightest galaxy in PSZ2 G126.57+51.61 (see below), was obtained at Sayan observatory 1.6-m telescope using the low and medium resolution spectrograph ADAM (Afanasiev et al., 2016; Burenin et al., 2016). In these observations the long slit position angle was set to obtain spectra of at least two red sequence galaxies in cluster, when possible. The 1-2 h total exposure was used at BTA 6-m telescope, and $6 \mathrm{~h}-$ at Sayan observatory 1.6-m telescope. The total exposure was split into 900-1200 s exposures, between the exposures the objects were shifted for 10-20" along the slit.

The $2 \mathrm{~d}$ spectra were bias and flat field corrected and transformed to single dispersion solution along the slit. The flat field and dispersion solution were calibrated using the spectra of calibration lamps obtained during every observation. The $2 \mathrm{~d}$ spectra obtained with SCORPIO instrument at BTA 6-m telescope were also corrected for fringes. For SCORPIO-2 instrument at BTA 6-m telescope and for ADAM spectrograph at Sayan observatory 1.6-m telescope these corrections are not necessary because these instruments are equipped with thicker deep depletion CCDs. After that $2 \mathrm{~d}$ spectra were aligned and combined, the cosmic rays were eliminated from the resulting $2 \mathrm{~d}$ spectrum. One dimensional object and background spectra were extracted in a standard way. Flux calibration of these spectra was done using the observations of stars from European Southern Observatory list of spectrophotometric standards. If needed, the spectra were divided by the normalized spectra of standard stars to correct for the atmospheric extinction lines.

All optical data reduction was done using $I R A F^{3}$ package, and also using our own software. In total, for these observations we used about $20 \mathrm{~h}$ of clear dark observing time at RTT150 telescope, about $9 \mathrm{~h}$ - at Sayan observatory 1.6-m telescope, about $3.6 \mathrm{~h}$ - at Calar Alto 3.5$\mathrm{m}$ telescope and about $14.5 \mathrm{~h}$ - at BTA 6-m telescope. Note, that much more observations of clusters from PSZ2 catalogue at lower redshifts were done at these telescopes. The results of these observations will be published in subsequent papers.

\section{THE RESULTS OF OBSERVATIONS}

In Fig. 2 the images from WISE survey in $3.4 \mu \mathrm{m}$ band are shown for every cluster (in the left panels). These images are cleaned from stars and convolved with $\beta$-model of $24^{\prime \prime}$ radius, which approximately corresponds to $180 \mathrm{kpc}$ linear size at redshift $z=0.8$. The right panels show the pseudocolor images of the clusters fields in SDSS irg filters which correspond to $R G B$ colors in these images. Images in filters $r i$ were taken from our observations (for some clusters we used the images in Bessel RI filters instead). The images in $g$ filter were taken from SDSS survey and

\footnotetext{
${ }^{3}$ http://iraf.noao.edu/
} 
PSZ2 G069.39+68.05
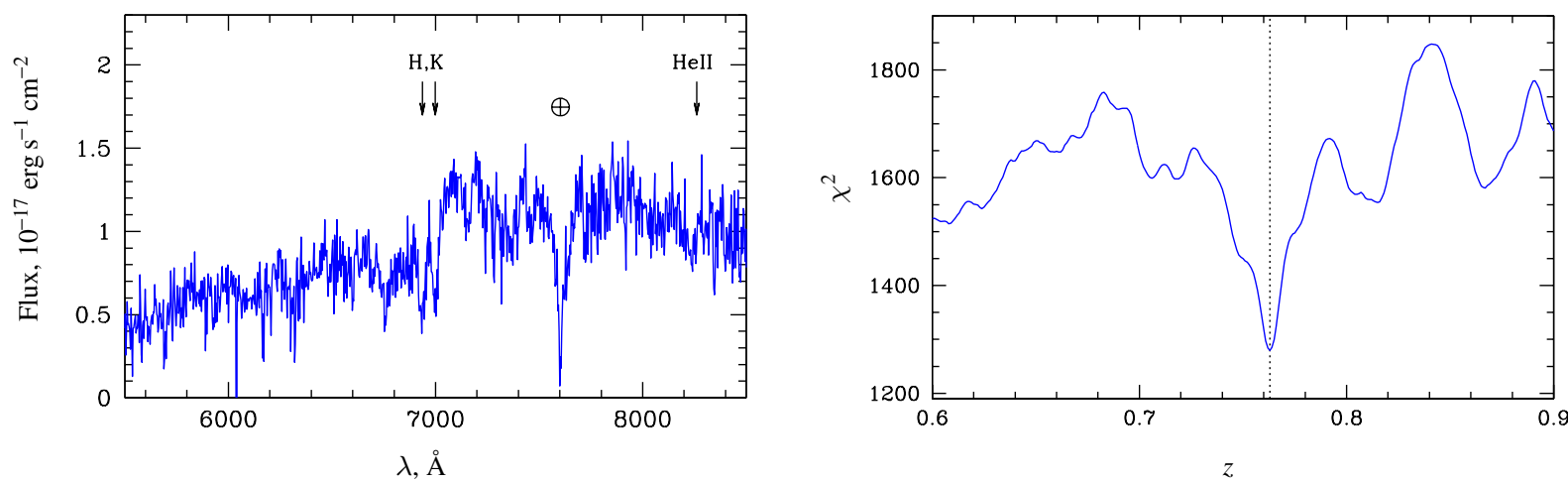

PSZ2 G092.69+59.92
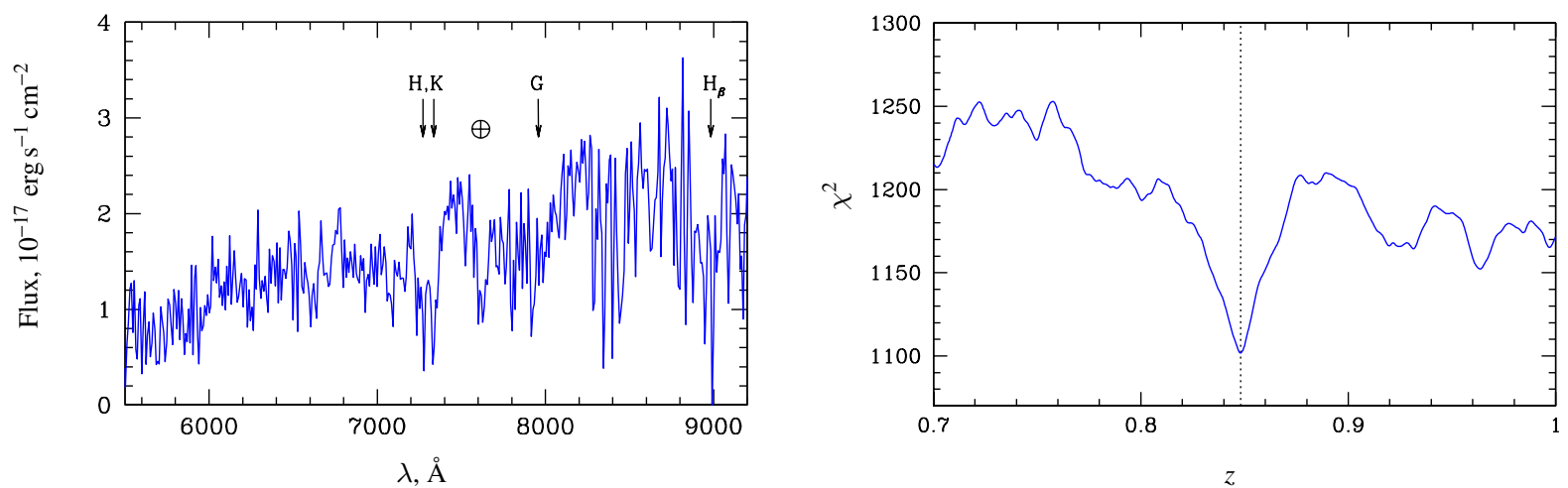

PSZ2 G126.28+65.62
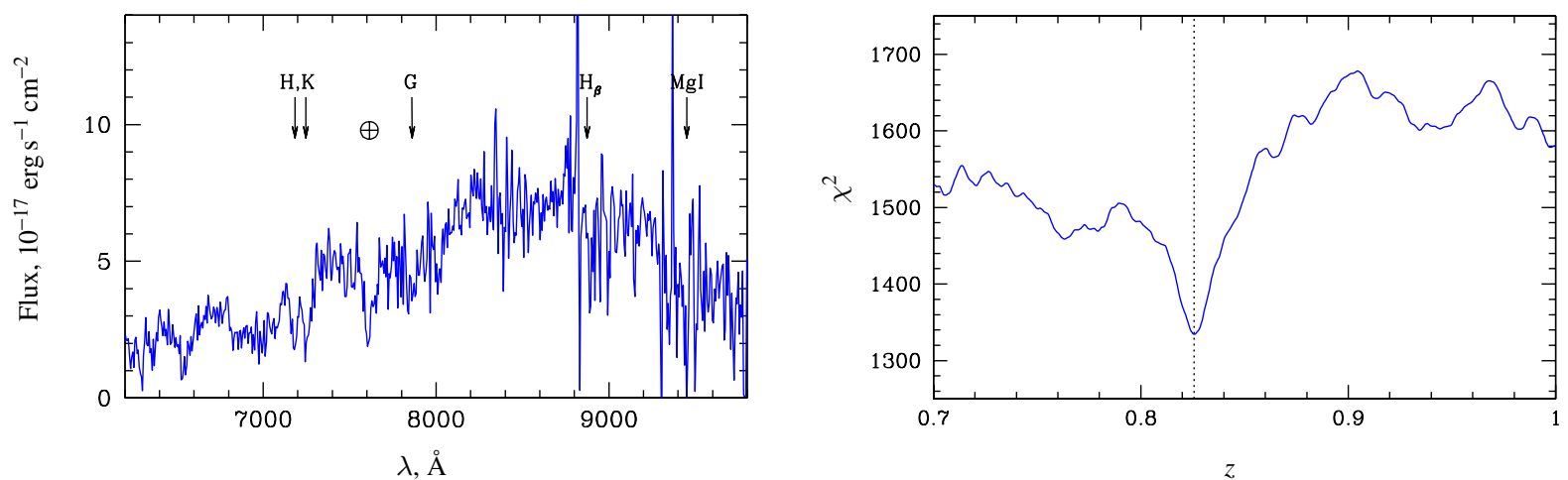

Fig. 3. The examples of galaxy cluster redshift measurements. Left panel: the spectrum of brightest cluster galaxy with some spectral features indicated, obtained at BTA 6-m telescope using SCORPIO and SCORPIO-2 spectrographs. Right panel: the value of $\chi^{2}$ obtained in result of cross correlation of this spectrum with the elliptical galaxy spectral template. 

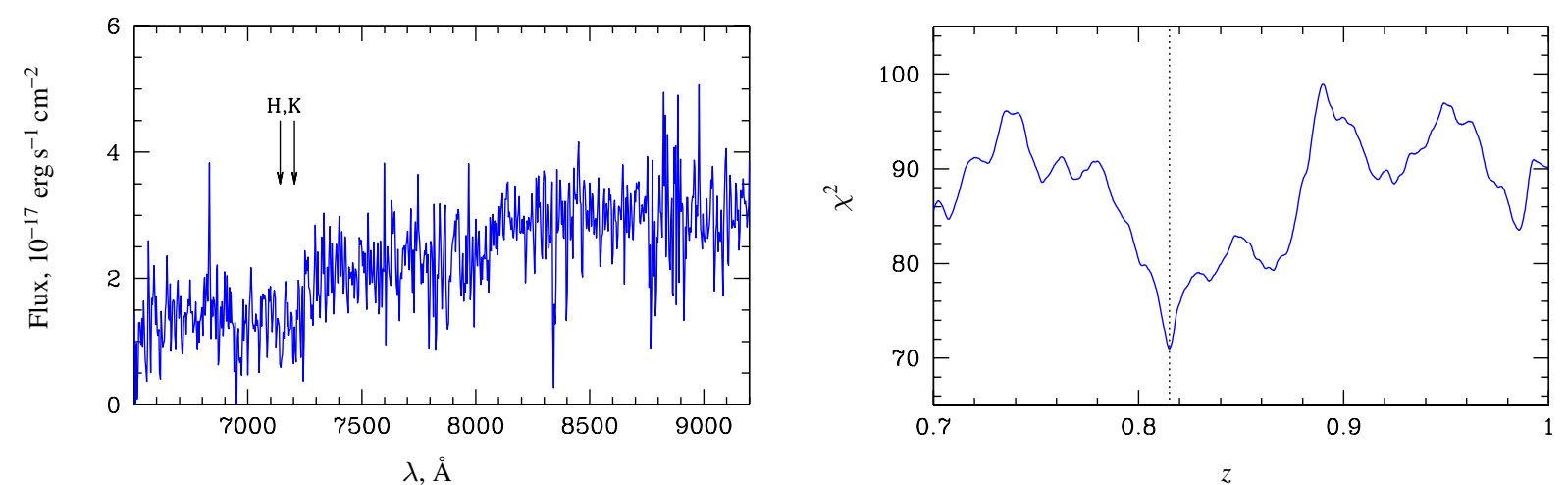

Fig. 4. Spectroscopic measurement of PSZ2 G126.57+51.61 galaxy cluster redshift. Left panel: spectrum of brightest cluster galaxy obtained at Sayan observatory 1.6-m telescope using ADAM spectrograph with $6 \mathrm{~h}$ total exposure. Right panel: the value of $\chi^{2}$ obtained in result of cross correlation of this spectrum with the spectral template of elliptical galaxy.

(or) Pan-STARRS1 survey. The centers of the images correspond to the clusters optical centers, the image size is $5^{\prime} \times 5^{\prime}$.

In the centers of the IR images shown in Fig. 2, the IR surface brightness excesses are observed, which indicates the presence of a large number of galaxies in this region of the sky. Note that for most objects discussed in this paper, i.e. for all objects except PSZ2 G092.69+59.92 and PSZ2 G237.68+57.83, the presence of increased surface density of the number of galaxies according to the data of WISE survey was mentioned in the PSZ2 catalogue. In pseudocolor images in Fig. 2 one can see that these galaxies are weak in the optical range and all of them are of about the same red color, i.e., they constitute the red sequence of the cluster. Using the colors of these galaxies, including data on color $i-z$ (see above), it is possible to obtain photometric redshift estimates, which appears to be higher than $z_{\text {phot }}=0.7$ for all objects. Spectroscopic redshifts were obtained for the brightest galaxies within the red sequences of the clusters.

In Fig. 3 the examples of spectroscopic measurements of the cluster redshifts are shown. The left panel shows the measured spectrum of the brightest cluster galaxies with some spectral features indicated, right panel - the $\chi^{2}$ value obtained in result of comparison of this spectrum with a template spectrum of an elliptical galaxy. The redshift measurement error is about $\delta z \approx 0.001$.

The redshift of galaxy cluster PSZ2 G126.57+51.61 was measured at Sayan observatory 1.6-m telescope using ADAM spectrograph. The brightest galaxy of this cluster is of magnitude $r^{\prime}=20.3$ and $i^{\prime}=18.9$, therefore to measure the redshift of this galaxy at small 1.6-m telescope the large exposure times were needed. We used the spectrum with $6 \mathrm{~h}$ total exposure, which was accumulated during the observations in Spring and Autumn 2017. The resulting spectrum and $\chi^{2}(z)$-redshift dependence are shown in Fig. 4. In this spectrum it is difficult to detect individual spectral lines, however, the $4000 \AA$ break located near the calcium
$H$ and $K$ lines is clearly visible, which allows to obtain a reliable measurement of the cluster redshift, $z=0.815$.

The optical identifications and spectroscopic redshift measurements for two clusters, PSZ2 G087.39-34.58 and PSZ2 G126.28+65.62, were obtained recently also at 10-m Gran Telescopio Canarias (GTC). The results of these measurements will be published later in separate paper. They are consistent with the results presented in this paper, but are of higher accuracy since the redshifts of larger number of galaxies are measured.

Four clusters PSZ2 G069.39+68.05, PSZ2 G087.3934.58, PSZ2 G092.69+59.92 and PSZ2 G126.28+65.62, were identified as distant galaxy clusters in the extended Planck SZ galaxy cluster catalogue (Burenin, 2017, B17). In this catalogue the redshifts for the clusters PSZ2 G069.39+68.05 and PSZ2 G087.39-34.58 were taken from SDSS survey. Although the redshifts of single galaxies at the side from the optical center of the cluster were used, these redshifts are confirmed by our measurements presented in this paper. In addition, in case of cluster PSZ2 G087.39-34.58, in the latest (14th) SDSS data release the redshift of central cluster galaxy was published, which agrees well with our measurement (see below). The cluster PSZ2 G092.69+59.92, apparently is a projection of two clusters, and the redshift of distant cluster, $z=0.848$, is also confirmed by the latest SDSS data. For cluster PSZ2 G126.28+65.62 the photometric redshift estimate was given in B17, which is also consistent with our measurements. The other clusters were not included in the B17 catalogue, because clusters with photometric redshift estimates above $z=0.7$ were not considered in B17.

The list of clusters that have been identified as a result of our work is presented in Table 1. The Table contains the coordinates of clusters optical centers, spectroscopic redshift measurements, the number of spectroscopically observed galaxies used to measure cluster redshift, also some notes on individual clusters are given. 
Table 1. High redshift galaxy clusters

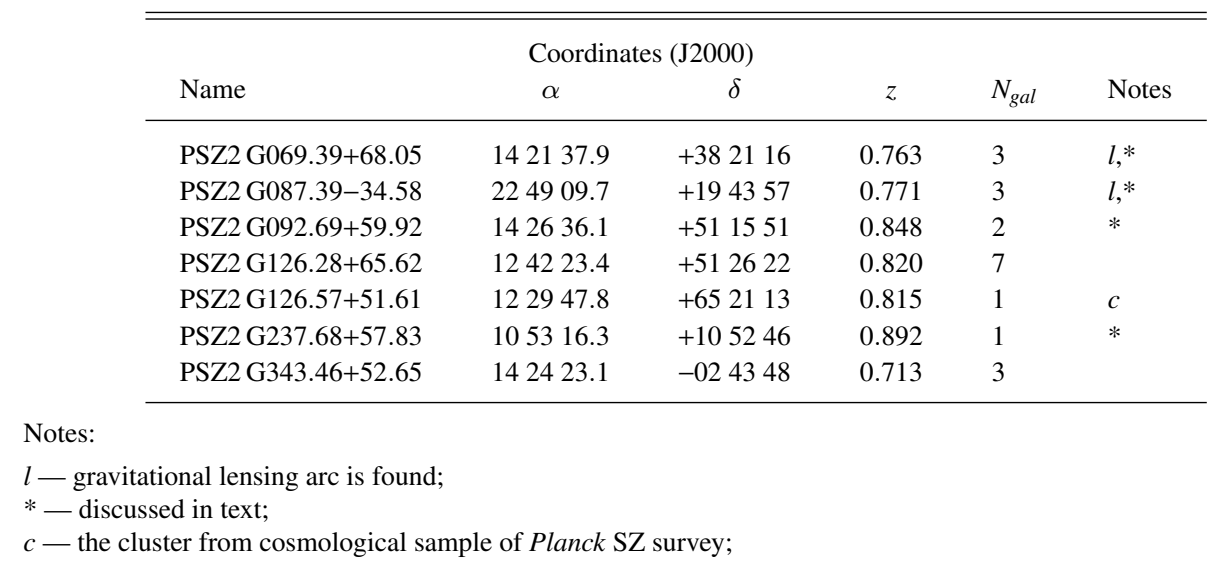

\section{Notes on individual objects}

\section{PSZ2 G069.39+68.05}

In the optical images of the central region of this cluster the gravitational lensing arc of 7 "length was found (see Fig. 5, left panel). In the spectrum of this galaxy that was obtained at the 6-m telescope BTA using SCORPIO-2 instrument with $3600 \mathrm{~s}$ exposure (Fig. 5, right panel) the absorption Lyman series hydrogen lines, as well as narrow absorption lines of ionized silicon, oxygen and carbon are detected. The redshift of the galaxy, measured from narrow absorption lines, is $z=4.262$. Note that in these observations the slit of the spectrograph was located across the direction of the lens, so only about $30 \%$ of light from lensed galaxy was observed inside the slit. The flux integrated over the entire area of the lens corresponds to stellar magnitude about 20.7 in $I$ band.

In SDSS survey data there is a spectrum of the galaxy located approximately $2^{\prime}$ to the North from the optical center of the cluster $(\alpha, \delta=14: 21: 39.9,+38: 23: 07, \mathbf{J} 2000)$. In 13th SDSS data release this spectrum was identified as a spectrum galaxy at $z=0.7617$, and in 14th data release as a spectrum of the quasar at $z=4.230$. Given that this object is extended and its color is consistent with the color of cluster red sequence, the first measurement is likely the correct one. It is consistent with out spectroscopic redshift measured from the spectra of galaxies indicated by arrows in Fig. 5. It is this SDSS redshift measurement was used earlier in the identification of this cluster presented in B17 catalogue.

\section{PSZ2 G087.39-34.58}

In the central part of the cluster the gravitational lensing arc of 14" length was found (see Fig. 6). The flux of lensed galaxy integrated over the entire area of the lens corresponds to the $i$ band magnitude about 21.7.

The galaxies, whose spectra were obtained at 6-m BTA telescope with SCORPIO spectrograph and then used to measure cluster redshift, are shown with arrows in Fig. 6. Note that in the latest (14th) SDSS data release there are two measurements of the redshifts for galaxies in this clusters that are consistent with our measurements. In B17 catalogue the redshift for this cluster redshift was measured using only one spectroscopic measurement from SDSS survey data available at that time. In Table 1 much more reliable redshift measurement for this cluster is provided, which was obtained using all the available data.

\section{PSZ2 G092.69+59.92}

This Sunyaev-Zeldovich source can be identified with the projection of two clusters of galaxies. One of them, located at redshift $z=0.463$, was discovered in the redMaPPer cluster survey (Rykoff et al., 2014). In the B17 catalogue this SZ source was automatically identified with that cluster because the clusters redshifts above $z \approx 0.7$ were not considered in this work. However, in the field of this SZ source there is also one more galaxy cluster, located at redshift $z=0.848$, the image of this cluster is shown in Fig. 2. Both clusters are clearly visible in the WISE image, which is shown in Fig. 7. The cluster at $z=0.848$ is found to be closer to the center of the SZ source and is of much higher IR luminosity. Therefore, most likely, it is this cluster is most massive one and provide the main contribution to the Sunyaev-Zeldovich signal of this Planck SZ source.

Note that for the galaxies of both clusters there are spectroscopic data in SDSS. For more distant cluster there is one spectroscopic measurement $(z=0.8421)$ in SDSS for the galaxy located at the approximately $3^{\prime}$ distance from the optical canter of the cluster in $N W$ direction. This measurement is in good agreement with our results.

\section{PSZ2 G237.68+57.83}

In the field of this Sunyaev-Zeldovich source it is possible to detect several clusters of galaxies, as shown in Fig. 8. Redshifts for clusters at $z=0.373$ and $z=0.355$ taken from 

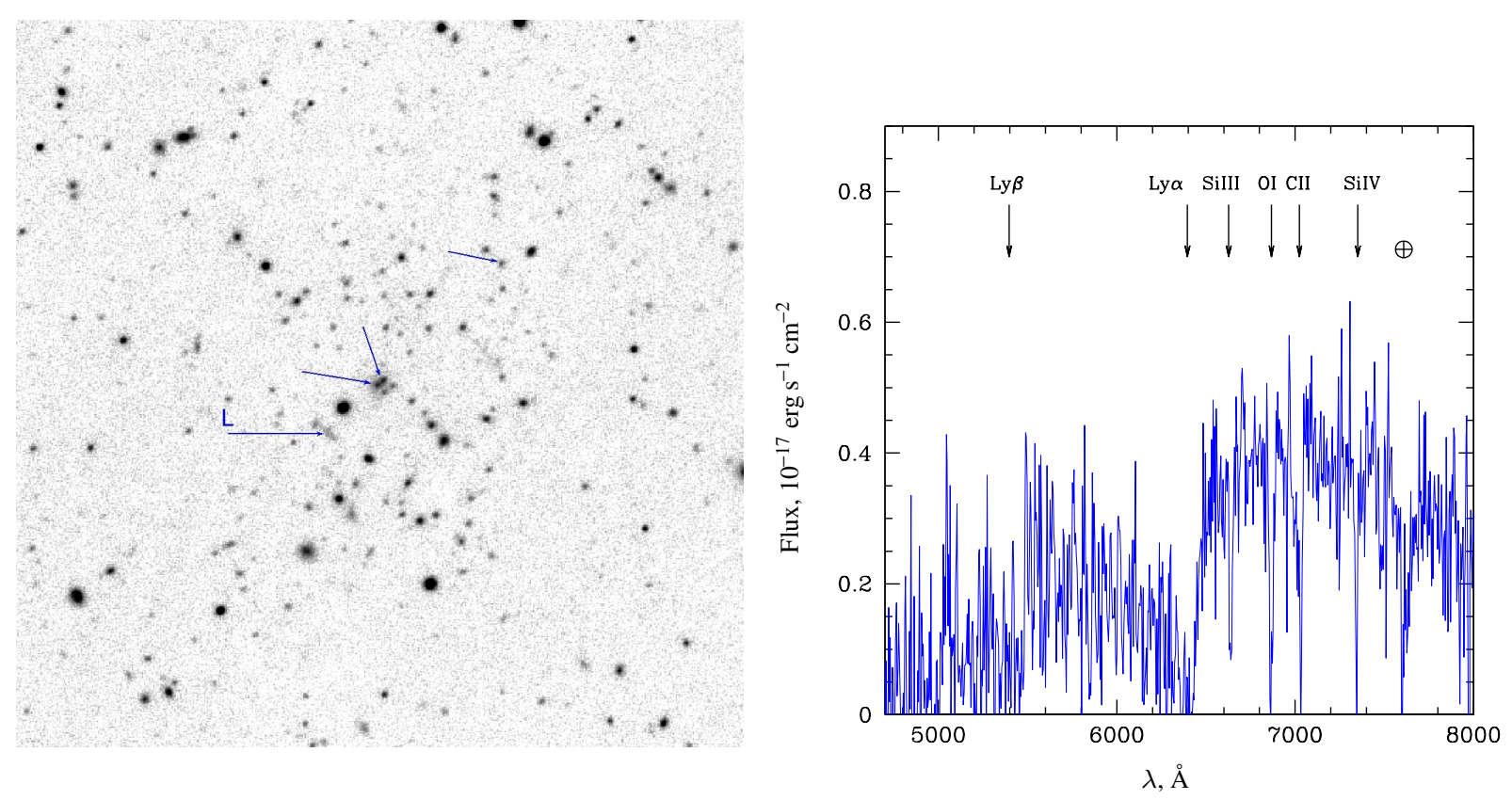

Fig. 5. Left panel: the image of the central part $\left(3^{\prime} \times 3^{\prime}\right)$ of the cluster PSZ2 G069.39+68.05 field in $I$ band, obtained with BTA 6-m telescope using SCORPIO-2 instrument with $150 \mathrm{~s}$ exposure. The objects with measured spectra are shown by arrows. The gravitational lensing arc is indicated by the letter $L$. Right panel: the spectrum of this arc, obtained at BTA 6-m telescope using SCORPIO-2 instrument with $3600 \mathrm{~s}$ exposure. Some spectral features observed at redshift $z=4.262$ are indicated.

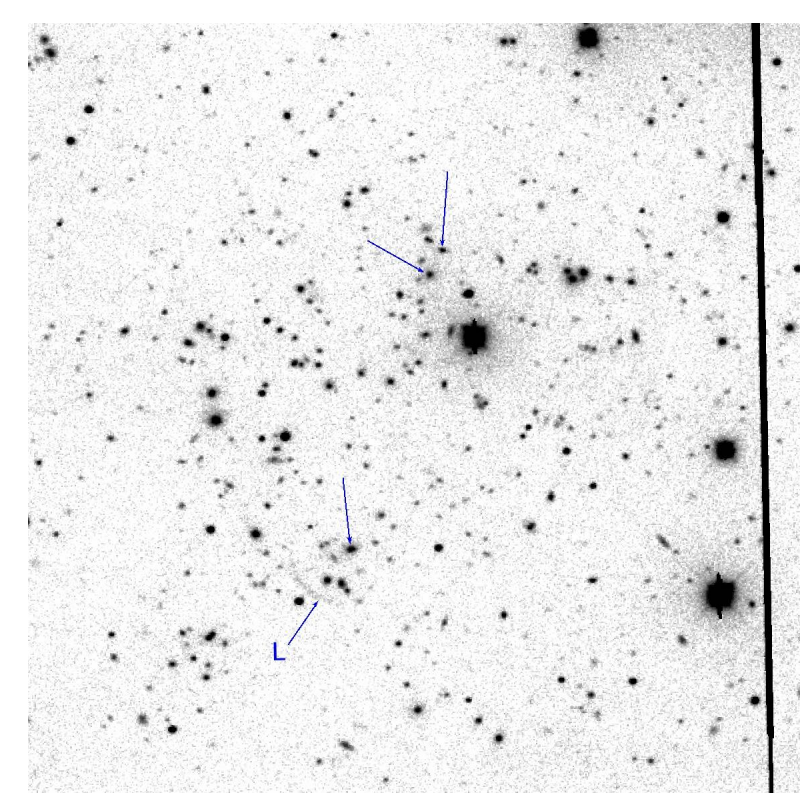

Fig. 6. The image of the central part $\left(3^{\prime} \times 3^{\prime}\right)$ of cluster PSZ2 G087.3934.58 field in $i$ band, obtained at Calar Alto 3.5-m telescope using MOSCA instrument with $900 \mathrm{~s}$ exposure. The galaxies, whose spectra were used to measure cluster redshift, are shown with arrows. The gravitational lensing arc is indicated by letter $L$.

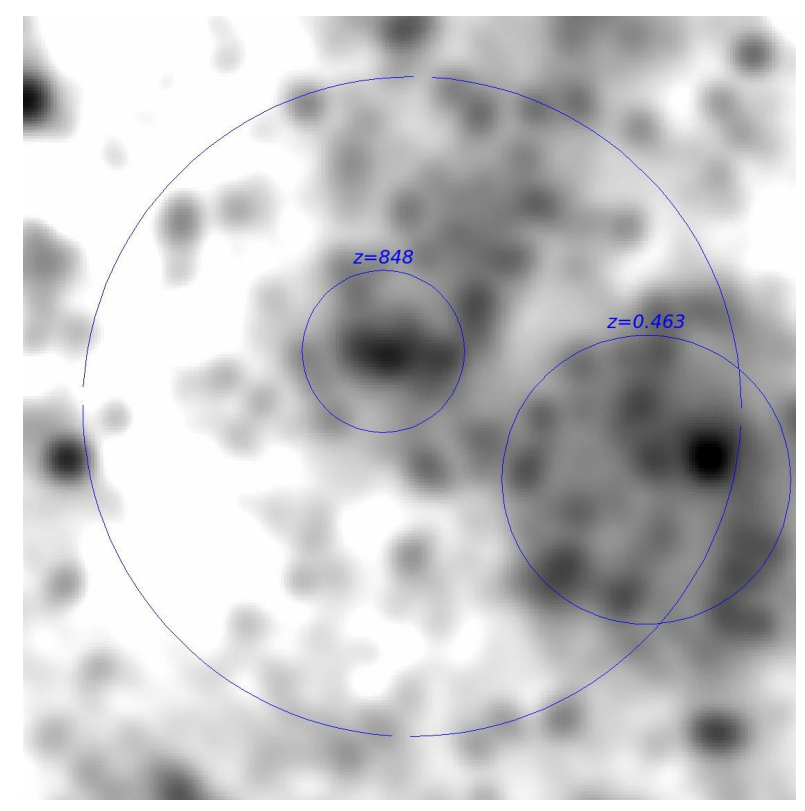

Fig. 7. The IR image of the field of cluster PSZ2 G092.69+59.92 from WISE survey, cleaned from stars and convolved with $\beta$-model of $24^{\prime \prime}$ radius. The localization of PSZ2 source is shown with a large circle $\left(5^{\prime}\right.$ radius). The circles of smaller radii show the clusters that are found in this field. 


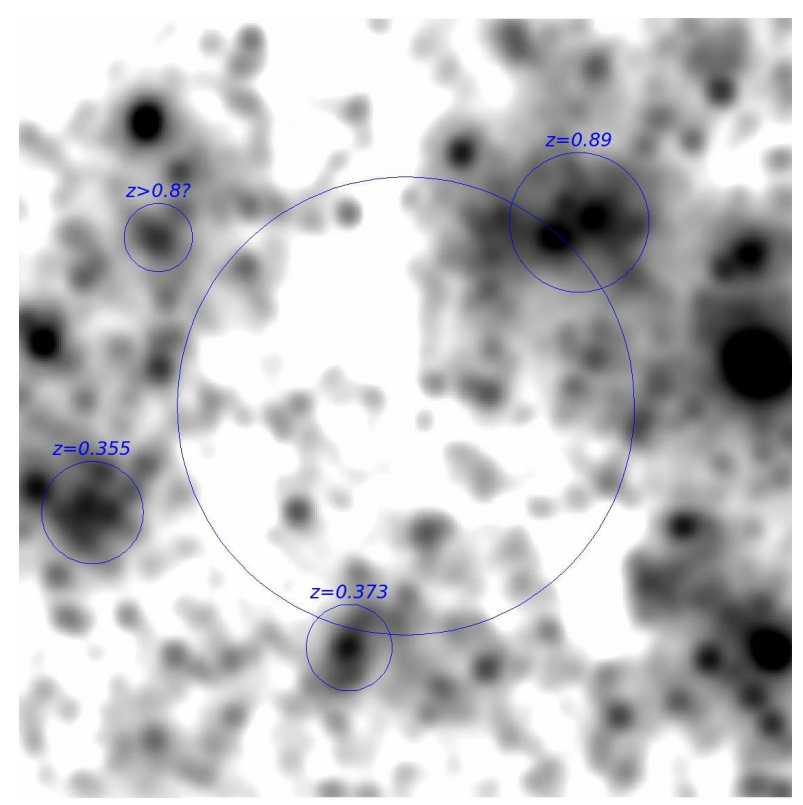

Fig. 8. The WISE image of the field of PSZ2 G237.68+57.83 SZ source, cleaned from stars and convolved with $\beta$-model of $24^{\prime \prime}$ radius. The localization of PSZ2 source is shown with large circle ( $5^{\prime}$ radius). The circles of smaller radii show the clusters which are found in this field. The remaining bright IR sources are connected with nearby galaxies in the field.

spectroscopic SDSS data. The cluster at $z=0.892$, apparently, is of the highest IR luminosity and therefore should be the most massive one and should give the largest contribution to the total Sunyaev-Zeldovich signal. However, other clusters may also give a significant contribution to the observed SZ signal. In order to measure the contribution of each cluster additional data in X-rays and (or) mm-band are required.

\section{CONCLUSIONS}

At the redshifts above $z \approx 0.5$ in 2 -nd Planck catalogue of Sunyaev-Zeldovich sources only the most massive clusters with masses above $M_{500} \approx 5 \cdot 10^{14} M_{\odot}$ are detected. These objects are extremely rare at high redshifts. For example, PSZ2 catalogue contains 12 clusters of galaxies at redshifts $z>0.7$, and 5 clusters - at redshifts $z>0.8$. In this paper we present the optical identifications and spectroscopic redshift measurements for 7 clusters of galaxies at redshifts $z>0.7$, of them 4 are found at redshifts $z>0.8$. The place of these clusters at the redshift-mass diagram is shown in Fig. 9. Here we use a simple $M_{500}$ mass estimate made using the Planck $y$-parameter map (Planck Collaboration, 2016a). Thus, one can see that the data presented in our work approximately double the number of known massive clusters in PSZ2 catalogue at high redshifts, $z \approx 0.8$.

In our list there are few more cluster candidates, some of them may be identified as distant clusters, located at redshifts $z \approx 0.8$ and higher in result of future observations. Observations of these objects are continued, however, we can already now state that in result of our work the com-

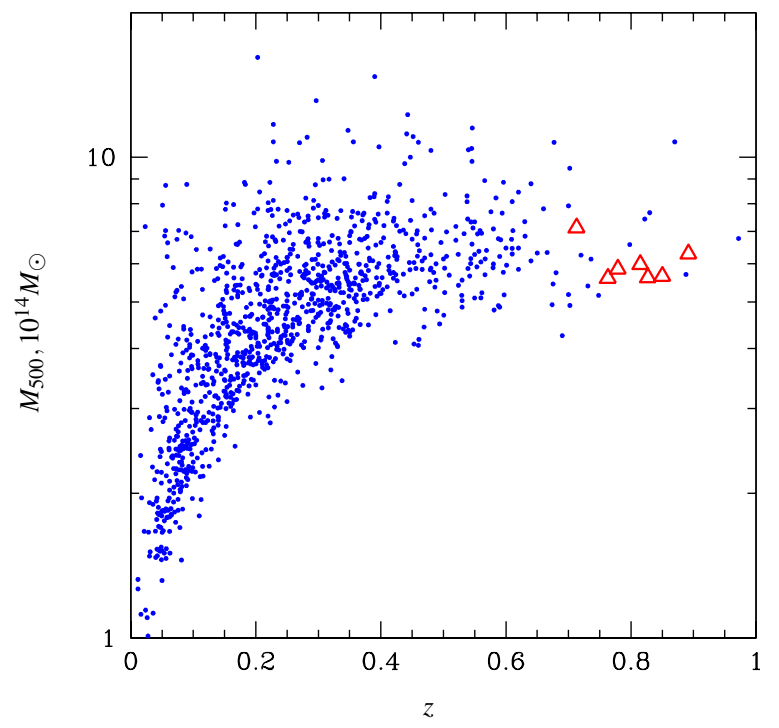

Fig. 9. The relations between redshifts and masses for clusters optically identified in PSZ2 catalogue (blue points) and in our work (red triangles).

pleteness of optical identification of SZ sources from PSZ2 catalogue for distant galaxy clusters in the Northern sky, at declination above $\delta>-20^{\circ}$, increased significantly. However, some number of distant clusters among the objects in PSZ2 catalogue should remain to be unidentified. In general, we can assume that among the Sunyaev-Zeldovich sources from PSZ2 catalogue there should be of order of 10 more unidentified clusters of galaxies at redshifts $z>0.7$. Therefore, some incompleteness of the PSZ2 catalogue for such clusters should remain.

We note that this incompleteness can not affect the cosmological results obtained with Planck survey galaxy cluster data. The reason is that the cosmological sample of PSZ2 catalogue is constructed using the clusters with higher SZ detection significance and these clusters are of even higher mass on average. The number of these even more massive clusters at high redshifts is very small. So, in cosmological sample of PSZ2 catalogue there are only three clusters at $z>0.7$. In our work we identified only one more such cluster, PSZ2 G126.57+51.61.

Our group continues the observations of galaxy clusters from the Planck all-sky Sunyaev-Zeldovich survey. In addition to the high-redshift clusters presented in this work, we were able to obtain optical identifications and to measure spectroscopic redshifts for a significant number clusters at lower redshifts. These results will be published in subsequent papers.

The work is supported by RSF grant 14-22-00271. In addition the authors are grateful to TUBITAK, IKI, KFU and AST for the support of the observations at RTT150 telescope. The work of IFB on the organization of a part of the observations at RTT150 telescope was partially funded 
by the subsidy 3.6714.2017/8.9 allocated to Kazan Federal University for the state assignment in the sphere of scientific activities. MVE is grateful to Basic Research Program P-7 of the Presidium of the Russian Academy of Sciences for partial support of the observations at 1.6-m Sayan observatory telescope, which is a part of "Angara" center.

\section{REFERENCES}

1. S. Amodeo, S. Mei, S.A. Stanford, C.R. Lawrence, J.G. Bartlett, D. Sterm et al., arXiv:1711.00021.

2. V.L. Afanasiev, A.V. Moiseev, Astronomy Letters 31, 194 (2005). [Pis'ma v Astron. Zhurn., 31, 214]

3. V.L. Afanasiev, A.V. Moiseev, Balt. Astr. 20, 363-370 (2011).

4. V.L. Afanasiev, S.N. Dodonov S.N., V.R. Amirkhanyan, A.V. Moiseev, Astrophys. Bull. 71, 479 (2016); Astrofizicheskij bulletin, 71, 514.

5. R.A. Burenin, A. Vikhlinin, A. Hornstrup, H. Ebeling, H. Quintana, A. Mescheryakov, Astrophys. J. Suppl. Ser. 172, 561 (2007).

6. R.A. Burenin and A.A. Vikhlinin, Astronomy Letters 38, 347 (2012). [Pis'ma v Astron. Zhurn., 38, 395]

7. R.A. Burenin, Astronomy Letters 41, 167 (2015) [Pis'ma v Astron. Zhurn., 41, 189].

8. R.A. Burenin, A.L. Amvrosov, M.V. Eselevich, V.M. Grigoryev, V.A. Arefiev, V.S. Vorobyev, et al., Astronomy Letters 42, 295 (2016) [Pis'ma v Astron. Zhurn., 41, 333].

9. R.A. Burenin, Astronomy Letters 43, 507 (2017) [Pis'ma v Astron. Zhurn., 43, 559].

10. G. Bruzual, S. Charlot, Mon. Not. R. Astron. Soc. 344, 1000 (2003).

11. A. Vikhlinin, R.A. Burenin, H. Ebeling, W.R. Forman, A. Hornstrup, C. Jones, A.V. Kravtsov, S.S. Murray, et al., Astrophys. J. 692, 1033 (2009a).

12. A. Vikhlinin, A.V. Kravtsov, R.A. Burenin, H. Ebeling, W.R. Forman, A. Hornstrup, C. Jones, S.S. Murray, et al., Astrophys. J. 692, 1060 (2009b).

13. V.S. Vorobiev, R.A. Burenin, I.F. Bikmaev, I.M. Khamitov, S.N. Dodonov, R.Ya. Zhuchkov, et al., Astronomy Letters 42, 63 (2016) [Pis'ma v Astron. Zhurn., 42, 81].

14. E.S. Rykoff, E. Rozo, M.T. Busha, C.E. Cunha, A. Finoguenov, A. Evrard, et al., Astrophys. J. 785, 104 (2014).

15. E.L. Wright, P.R.M. Eisenhardt,, A.K. Mainzer, M.E. Ressler, R.M. Cutri, T. Jarrett, J.D. Kirkpatrick, D. Padgett, et al., Astron. J. 140, 1868 (2010).

16. Planck Collaboration, Planck 2013 Results XX: P.A.R. Ade, N. Aghanim, C. Armitage-Caplan, et al., Astron. Astrophys. 571, A20 (2014a).

17. Planck Collaboration, Planck 2013 Results XXIX: P.A.R. Ade, N. Aghanim, C. Armitage-Caplan, et al., Astron. Astrophys. 571, A29 (2014b); arXiv:1303.5089.

18. Planck Collaboration, Planck Intemediate Results XXVI: P.A.R. Ade, N. Aghanim, M. Arnaud et al., Astron. Astrophys. 582, A29 (2015a); arXiv:1407.6663.
19. Planck Collaboration, Planck 2013 Results XXXII: P.A.R. Ade, N. Aghanim, C. Armitage-Caplan, et al., Astron. Astrophys. 581, A14 (2015b); arXiv:1502.00543.

20. Planck Collaboration, Planck 2015 Results XXIV: N. Aghanim, M. Arnaud, M. Ashdown, et al., Astron. Astrophys. 594, A22 (2016a) ; arXiv:1502.01596.

21. Planck Collaboration, Planck 2015 Results XXIV: P.A.R. Ade, N. Aghanim, M. Arnaud et al., Astron. Astrophys. 594, A24 (2016b) ; arXiv:1502.01597.

22. Planck Collaboration, Planck 2015 Results XXVII: P.A.R. Ade, N. Aghanim, M. Arnaud et al., Astron. Astrophys. 594, A27 (2016c); arXiv:1502.01598.

23. Planck Collaboration, Planck Intermediate Results XXXVI: P.A.R. Ade, N. Aghanim, M. Arnaud et al., Astron. Astrophys. 586, A139 (2016d); arXiv:1504.04583.

24. SDSS Collaboration: F.D. Albareti, C.A. Prieto, A. Almeida, et al., Astrophys. J. Suppl. Ser., in press (2017); arXiv:1608.02013.

25. R.A. Sunyaev and Ya.B. Zeldovich, Comments on Astrophysics and Space Physics, 4, 173 (1972).

26. K.C. Chambers, E.A. Magnier, N. Metcalfe, H.A. Flewelling, M.E. Huber, C.Z. et al., arXiv:1612.05560 (2016). 